\title{
Idiopathic intracranial hypertension or pseudotumour cerebri? The conundrum of nomenclature in the presence of normal cerebrospinal fluid pressures
}

\author{
P. Magesh ${ }^{1}$, A. Sendil Kumar ${ }^{2}$, K. Prabhuraman ${ }^{3, *}$ \\ ${ }^{\mathbf{1}}$ Assistant Professor, ${ }^{\mathbf{2 , 3}}$ Associate Professor, Dept. of Neurosurgery, ${ }^{\mathbf{1}, \mathbf{3}}$ Madras Medical College, Chennai, Tamil Nadu, ${ }^{\mathbf{G}}$ Government \\ Vellore Medical College, Vellore, Tamil Nadu, India \\ *Corresponding Author: K. Prabhuraman \\ Email: drprabhuraman@yahoo.com
}

\begin{abstract}
Idiopathic Intracranial Hypertension (IIH), also known as Pseudo tumour cerebri (PTC), is a condition in which the patient presents with signs and symptoms of increased intracranial pressure, with no demonstrable organic cause in the brain imaging including venous sinus thrombosis, and with normal cerebrospinal fluid (CSF) composition. Atypical cases of IIH have been reported periodically, and an important subset of these patients have normal CSF Opening pressure (OP) on lumbar puncture (LP). Application of strict criteria for diagnosing such patients needs reconsideration.

We present 7 cases of IIH with normal CSF Opening pressures, out of 30 consecutive patients of suspected IIH admitted to the neurosurgery ward of a tertiary care hospital over a period of 2 years, kindling interest in nomenclature and diagnostic dilemma if strict criteria are adhered to.
\end{abstract}

Keywords: Idiopathic intracranial hypertension (IIH), Pseudo tumour cerebri (PTC), Opening pressure, Lumbar puncture, Normal CSF pressures.

\section{Introduction}

Idiopathic Intracranial Hypertension (IIH), also known as Pseudo tumour cerebri (PTC), is a condition in which the patient presents with signs and symptoms of increased intracranial pressure (ICP), with no demonstrable organic cause in the brain imaging including venous sinus thrombosis, and with normal cerebrospinal fluid (CSF) composition. Dandy was the first to methodically describe the symptoms of raised ICP, document CSF pressures and composition and rule out the possibility of brain tumours with ventriculography in his description of this condition in a set of 22 patients in $1937 .{ }^{1}$

The Dandy criteria for the diagnosis of IIH/PTC, modified by Smith et al., in 1985, are: (a) signs and symptoms of increased intracranial pressure; (b) absence of localized findings on neurologic examination; (c) normal neuroradiologic studies; (d) awake and alert patient; (e) no other cause of increased intracranial pressure present and are being widely followed as modified Dandy criteria. ${ }^{2}$

The classical features of IIH headache described by the International Headache Society (ICHD III) are: New headache, or a significant worsening of a pre-existing headache, caused by and accompanied by other symptoms and/or clinical and/or neuro-imaging signs of IIH, with typical features suggestive of IIH. ${ }^{3}$ Diagnostic criteria:

A. New headache, or a significant worsening of a preexisting headache, fulfilling criterion $\mathrm{C}$

B. Both of the following:

1. IIH has been diagnosed

2. CSF pressure exceeds $250 \mathrm{~mm} \mathrm{CSF}$ (or $280 \mathrm{~mm} \mathrm{CSF}$ in obese children)

C. Either or both of the following:

1. Headache has developed or significantly worsened in temporal relation to the IIH, or led to its discovery
2. Headache is accompanied by either or both of the following:

\section{a. Pulsatile tinnitus}

b. Papilloedema

There are reports of atypical cases of IIH, and include those with headaches that are not classical for ICP and those without visual disturbances. Some patients have had symptoms suggestive of IIH, without papilloedema, but have demonstrable increase in CSF opening pressure, responding to pressure reducing agents, and have since been designated as IIH without papilloedema. ${ }^{4}$

Johnston et al. called attention to some cases that bear a close resemblance to PTC but fail to comply with one or more of the accepted diagnostic criteria. ${ }^{5}$ These authors proposed that the concept of PTC be broadened to include other atypical forms. One of these atypical forms was that of "normal pressure pseudotumor syndrome", and is being reported periodically.

\section{Objective}

To draw attention to the paradox in nomenclature of patients presenting with typical signs and symptoms of raised ICP with normal Lumbar CSF opening pressures - to name them IIH even when there is no measured evidence of hypertension or simply normal pressure PTC?, and to record the therapeutic effect of a single LP.

\section{Materials and Methods}

This study was conducted on 30 consecutive cases of suspected IIH/PTC admitted in the neurosurgery department at the Institute of Neurosurgery, Madras Medical College and Rajiv Gandhi Government General Hospital, Chennai 3, between 2008 and 2010 with a clinical diagnosis of IIH, as suggested by the 'Modified Dandy criteria'. The approval of the Institutional Ethics Committee was obtained. 
All patients had headache of varying severity, persistent and constant for few weeks prior to admission, necessitating medical advice. The temporal profile of headache was suggestive of raised intracranial pressure in all of them. All patients had bilateral papilloedema clinically, and were confirmed by the Neuro-ophthalmologist of the Institute, and visual field charting was done with Goldmann perimetry. None had any focal neurological deficit after a thorough neurological examination, other than lateral rectus palsy in a few. None had history of chronic medications. All patients were alert and co-operative. All had CT brain and MRI Brain and MRV done to rule out venous sinus thrombosis and there was no evidence of hydrocephalus, mass or structural or vascular lesions in any of them.

All patients were explained in detail about the procedure and written informed consent was obtained for performing lumbar puncture under local anaesthesia. Each patient was positioned in the right lateral decubitus position and under sterile aseptic precautions, lumbar puncture was performed with $20 \mathrm{G}$ spinal needle under local anaesthesia. The needle was connected to a saline manometer without letting out any CSF. The patient was allowed to relax, extend lower limbs and neck, lying comfortably in the right lateral position, and the opening pressure was noted in centimetres of water (CSF) after the saline column stabilised.

About 20 to $25 \mathrm{ml}$ of guarded therapeutic drainage of CSF was done in each case. Cytological and biochemical analysis of the CSF samples were found to be normal in all cases. The procedure was uneventful in all cases, and the patients tolerated well.

\section{Results}

There were 8 males and 22 females in all, with age ranging from 15 to 49 years. The mean CSF OP was 36.1 $\mathrm{cmH} 2 \mathrm{O}$ ranging from 14 to $83 \mathrm{cmH} 2 \mathrm{O}$. Seven patients, 3 males and 4 females, had OP below $25 \mathrm{cmH} 2 \mathrm{O}$, ranging from 14 to $21 \mathrm{cmH} 2 \mathrm{O}$, and did not meet the typical modified Dandy criteria for a diagnosis of IIH/PTC. Of these, five patients had OP less than $20 \mathrm{cmH} 2 \mathrm{O}$ and two had $21 \mathrm{cmH} 2 \mathrm{O}$.The observations and clinical features of these seven patients are summarized in table 1 . All patients were advised complete bed rest for 24 hours following LP, and were started on $\mathrm{T}$. Acetazolamide $1 \mathrm{gm} / \mathrm{day}$ in divided doses. They were encouraged to take plenty of oral fluids. Dietary advice was given to prevent hypokalemia secondary to the drug.

All patients reported subjective improvement on the day following LP, and headache and visual blurring were the first symptoms to be relieved. None reported new headache suggestive of low tension.

All patients with OP below $25 \mathrm{cmH} 2 \mathrm{O}$ were given the option of repeat LP and OP estimation in the next two days, but they refused, apparently due to the significant subjective improvement in symptoms. All but one patient did well with conservative management.

One patient had transient improvement for about a week following LP, but had persistent papilloedema and enlargement of blind spot on field charting. She also reported recurrence of double vision and headache. Second LP done on her was also below $20 \mathrm{cmH} 2 \mathrm{O}$. She underwent lumbo-peritoneal shunt, and improved.

\section{Discussion}

Demonstration of Raised ICP: The diagnosis of IIH or PTC, by definition and convention requires proof of increased ICP. The study by Lenfeldt et al., demonstrated the accuracy of the lumbar puncture technique to determine ICP in both absolute and relative terms. ${ }^{7}$ In the absence of any pathological obstruction in the CSF pathway, the measurement of LP OP reflects ICP.

Modified Dandy criteria requires presence of LP OP of more than $25 \mathrm{cmH} 2 \mathrm{O}$ to confirm a diagnosis of PTC. ICHD III criteria mandates CSF pressures in adults to be more than $25 \mathrm{cmH} 2 \mathrm{O}$ and in obese children to be more than $28 \mathrm{cmH} 2 \mathrm{O}$.

Our series had 7 patients with OP below the criteria of $25 \mathrm{cmH} 2 \mathrm{O}$, but had all other symptoms and signs of raised ICP. They responded favourably after a therapeutic drainage of CSF, and Acetazolamide, confirming the presence of IIH/PTC.

Single or Repeat Lumbar Puncture: Normal pressure PTC has been reported periodically and perhaps the first such description was by Johnston. ${ }^{5}$ A series of six such patients was reported by Abdelfatah. ${ }^{8}$ Such cases have also been reported by Green, Biousse, and Sou Youn Suh. ${ }^{6,9,10}$

Johnston et al., reported a series of atypical IIH patients, one of which was a 13 year old boy with disc edema and visual field defects, that resolved following lumbo-peritoneal shunt insertion even though his CSF pressures were normal on single LP and with continuous ICP monitoring later. ${ }^{5}$ Green et al., reported the case of an 18 year old woman IIH patient with normal pressures measured on three occasions, with documented improvement in symptoms following LP and ultimately benefitted with optic nerve sheath fenestration. ${ }^{6}$ Biousse described a 31 year old woman with papilloedema and clinical profile fitting IIH, but with normal LP OP, who improved following LP and continued to do better with conservative management. ${ }^{9}$ Soh YS reported two female patients with clinical findings of IIH but with pressures below $25 \mathrm{cmH} 2 \mathrm{O}$, demonstrated twice in one, and both patients did well after LP and were managed conservatively ${ }^{10}$. The study by Abdelfatah describes 6 obese anaemic female patients, two of whom were pregnant, and four had history of excess vitamin A intake and or on hormonal contraceptive. All patients had papilloedema, underwent LP twice, with mean OP recorded to be 11 $\mathrm{cmH} 2 \mathrm{O}$, falling to $7.3 \mathrm{cmH} 2 \mathrm{O}$ respectively, and all had responded favourably to medical management. ${ }^{8}$

A repeat LP or continuous monitoring of ICP for 24 hours may be required in appropriate clinical settings for confirmation of IIH as recommended by Friedman and Jacobson, but all but one of our patients refused a second tap. ${ }^{11}$

Therapeutic Advantage of Single LP: The explanation for the observed subjective and satisfactory relief of symptoms 
following a single LP in these patients is given by Simone et al. ${ }^{12}$ As the CSF production rate allows its complete turnover up to four times in 24 hours, the longstanding changes observed cannot be easily considered a long term effect of fluid removal. Actually, a single lumbar puncture could act as a switch between two different balance states venous and CSF pressures. Although secondary to CSF hypertension, venous sinuses may have an important role in hypertensive status maintenance. They go on to hypothesise that, at least in IIH patients with a history of longstanding remissions after a single or a few serial lumbar punctures, a cryptogenetic transient increase of CSF pressure can induce a sinus compression.

The treatment of raised ICP itself begins with the diagnostic lumbar puncture, which is often effective in transiently improving symptoms and signs. Interestingly, it is not uncommon to observe a lasting clinical remission following a single lumbar puncture in some IIH patients, obviating the need for further medical or surgical treatment. This phenomenon cannot be simply explained by the amount of CSF drained, or by the hole made in the dura by the needle used for the lumbar puncture. Interaction of various factors, such as CSF formation, compliance, cerebral blood flow, and outflow resistance, leading to multiple stable and unstable equilibrium levels of ICP in IIH is the explanation proposed by Mathematical models of CSF hydraulics. ${ }^{13}$ If a single lumbar puncture lowers the CSF pressure beyond the threshold level of an unstable equilibrium, the ICP must settle into a lower stable pressure state until other factors cause the pressure to exceed that threshold. This observation emphasizes the importance of the initial lumbar puncture as a therapeutic procedure in IIH in addition to its diagnostic importance. It also explains why some patients dramatically improve after two or three lumbar punctures or require episodic lumbar punctures to remain asymptomatic. ${ }^{14}$

In majority of patients, as in our series, diagnostic LP proves to be therapeutic, and medical management for a variable period of time, as per clinical response are often sufficient for a remission. All patients in our series had shown improvement with a single LP, establishing its definitive therapeutic role. All but one patient in the normal pressure group continued to do well following LP and medical management. We believe that the dural hole made by the $20 \mathrm{G}$ needle is kept patent by the relatively higher pressure on the other side of the dura, and, in effect it functions technically as a shunt, diverting the CSF from the subarachnoid space to the extradural space till the pressures equilibrate.

Mechanisms / Pathophysiology: Johnston et al. proposed two possible explanations for the presence of papilledema in the absence of measured elevated ICP. ${ }^{5}$ Firstly, it was proposed that the situation could be likened to normalpressure hydrocephalus, in which there is a definite abnormality of CSF circulation and volume relieved by drainage, without a demonstrable abnormality of CSF pressure. Secondly, it was hypothesized that local abnormalities in the region of the optic nerve sheath are responsible for the development of papilledema, with relatively normal ICP allowing a local build up of pressure that is not reflected in the pressure measurement elsewhere in the subarachnoid space.

Another possible explanation by Suh SY suggests that some patients may have optic discs that are more susceptible to lower ICP than others. ${ }^{10}$

As has been observed by Abdulfetah et al., the cause of the normal CSF pressure at LP in patients with normal pressure PTC despite the symptoms and signs suggestive of increased ICP is obscure. The presence of papilledema in these patients indicates increased ICP, which due to a certain cause failed to be transmitted to the spinal subarachnoid space. ${ }^{8}$

\section{Are low pressures and PTC mutually exclusive? Or can it still be PTC?}

Corbett has observed that the diagnosis of PTC should not be made under any circumstances without finding elevated spinal fluid pressure. ${ }^{15}$ Normal intracranial pressure has been defined as $136 \mathrm{~mm} \mathrm{H} 20(\mathrm{SD} \pm 37.6)$ in patients of normal weight, and $167 \mathrm{~mm} \mathrm{H} 2 \mathrm{O}$ (SD \pm 36.46$)$ in obese patients. Johnston and Paterson have observed wide fluctuations of CSF pressures. ${ }^{16}$ It has also been noted that OP may vary in the same individual when measured at different times.

Mollan et al., have observed that despite optimising the conditions for lumbar puncture, OP is still a one-off reading. ${ }^{17}$ It is, however, useful retrospectively to ask the patient if their symptoms of visual obscurations, headache, etc., improved for a few days after the lumbar puncture. Improvement supports the presence of raised intracranial pressure.

Tibussek et al., have concluded that OP measured during an LP is a snapshot of a highly dynamic process and that the results of opening pressure measurement must be correlated with caution and in the overall context of the patient's clinical history, symptoms and clinical signs. ${ }^{18}$

Nevertheless, repeat LP and 24-hour monitoring of ICP and reassessment of OP may not be possible in all situations due to patient preference and logistic limitations.

\section{Nomenclature - What's in a name?}

The diagnostic criteria for pseudotumor cerebri syndrome (PTCS) have been revised recently by Friedman et al. ${ }^{19}$ The authors mention "probable PTCS" as a subgroup of PTCS for patients with an OP even below $20 \mathrm{~cm} \mathrm{H} 2 \mathrm{O}$ but with clinical signs and symptoms suggestive of PTCS. While demonstration of raised CSF pressure is clearly an important component of the diagnosis of PTCS and can, in most instances be satisfactorily achieved by manometry via lumbar puncture, there will be cases with genuine PTCS in whom a single manometric pressure measurement on one or more occasions will be normal. This will be due either to the measurement being carried out during a trough in the ICP level (most instances) or to genuine 'normal-pressure' PTCS in a small but unknown proportion of cases. In these revised criteria, documentation of an elevated CSF opening pressure 
is required for the diagnosis of definite PTCS, but the diagnosis of probable PTCS may still be made in an otherwise typical patient if bilateral papilledema is present and the measured opening pressure is not elevated.

Distelmaier et al. have proposed the concept of "probable IIH", and suggest adding 1) CSF OP may be less than $20 \mathrm{cmH} 2 \mathrm{O}$ and 2) clear clinical response to initial pressure release and/or treatment to the diagnostic criteria. ${ }^{20}$

It has been suggested that ICHD criteria need reconsideration in view of the fact that among a cohort of patients with headache secondary to IIH, no patient showed the elevated levels of opening CSF pressure established as a criteria for the disorder. ${ }^{21}$

ICHD 3 recognises that "Relief of the headache after CSF removal is supportive of the diagnosis but not on its own diagnostic", but is still far from approving it as a significant criterion. ${ }^{3}$

Hence, irrespective of the term used for diagnosis, it is prudent to recognise the fact that cases of IIH with normal pressures or Normal pressure PTC do occur, and early identification goes to save the patient of morbidity and avoidable complications.

Table 1: Demographic data, visual parameters and modalities of management of 7 patients with Idiopathic Intracranial Hypertension

\begin{tabular}{|c|c|c|c|c|c|c|c|c|}
\hline S. No. & Age & Sex & OP & Visual Field & 6th CN Palsy & Papilloedema & Obesity & Management \\
\hline 1 & 28 & $\mathrm{~F}$ & 21 & $\mathrm{BS} \uparrow$ & - & + & - & Conservative \\
\hline 2 & 40 & $\mathrm{~F}$ & 18 & $\mathrm{BS} \uparrow$ & - & + & + & Conservative \\
\hline 3 & 37 & $\mathrm{~F}$ & 19 & $\mathrm{BS} \uparrow$ & + & + & + & LP Shunt \\
\hline 4 & 36 & $\mathrm{M}$ & 16 & $\mathrm{BS} \uparrow$ & - & + & Conservative \\
\hline 5 & 45 & $\mathrm{M}$ & 14 & $\mathrm{BS} \uparrow$ & - & + & Conservative \\
\hline 6 & 49 & $\mathrm{M}$ & 21 & $\mathrm{BS} \uparrow$ & - & + & Conservative \\
\hline 7 & 15 & $\mathrm{~F}$ & 19.5 & - & - & + & Conservative \\
\hline $\mathrm{OP}$ & \multicolumn{8}{|l|}{} \\
\hline $\mathrm{BS} \uparrow$ & \multicolumn{8}{|l|}{} \\
\hline
\end{tabular}

\section{Conclusion}

Strict reliance on criteria on OP in patients with papilloedema and other symptoms and signs of raised ICP may lead to avoidable delays in diagnosis and initiation of treatment. As papilledema is reversible with appropriate treatment in IIH patients, and untreated papilledema can result in progressive and irreversible visual loss, a broader approach ends diagnostic dilemma.

With many atypical cases being reported, patients with normal CSF OP may be categorised as "Atypical IIH or Normal pressure PTC or Probable PTC", and the diagnostic criteria may be modified to accommodate such cases. A single diagnostic LP, while recording the OP, doubles up as therapy in a significant proportion of IIH/PTC patients.

\section{References}

1. Dandy WE.Intracranial pressure without brain tumour; diagnosis and treatment. Ann Surg 1937;106:492-513.

2. Smith JL.Whence pseudotumor cerebri? J Clin Neuroophthalmol 1985;5:55-56.

3. Headache Classification Committee of the International Headache Society (IHS) The International Classification of Headache Disorders, 3rd edition. Cephalalgia 2018;38(1):1211

4. Marcelis J and Silberstein SD. Idiopathic intracranial hypertension without papilledema. Arch Neurol 199;48:392399.

5. Johnston I, Hawke S, Hamalgyi M, Teo C.The Pseudotumour Syndrome:disorders of CSF circulation causing intracranial hypertension without ventriculomegaly. Arch Neurol 1991;48:740-747.

6. Green JP, Newman NJ, Stowe ZN, Nemeroff CB. "Normal pressure" pseudotumor cerebri. J Neuro-ophthalmol 1996; 16:241-246.
7. Lenfeldt N, Koskinen LOD, Bergenheim AT, Malm J, Eklund A.CSF pressure assessed by lumbar puncture agrees with intracranial pressure. Neurol 2007;68:155-158.

8. Abdelfatah MA. Normal Pressure Pseudotumor Cerebri: A Series of Six Patients. Turk Neurosurg 2017;27(2):208-211.

9. Biousse V, Bousser MG, Schaison M. Normal pressure pseudotumor cerebri. J Neuroophthalmol 1997;17(4):279-280.

10. Suh SY, Kim SJ: IIH with normal CSF pressures? Indian J Ophthalmol 2013;61(11):681-682.

11. Friedman DI, Jacobson DM. Diagnostic criteria for idiopathic intracranial hypertension. Neurol 2002;59:1492-1495.

12. De Simone R, Marano E, Fiorillo C, Briganti F, Salle FD, Volpe A et al. Sudden re-opening of collapsed transverse sinuses and longstanding clinical remission after a single lumbar puncture in a case of idiopathic intracranial hypertension. Pathogenetic implications. Neurol Sci 2005;25:342-344.

13. Bateman GA, Stevens SA, Stimpson J.A mathematical model of idiopathic intracranial hypertension incorporating increased arterial inflow and variable venous outflow collapsibility. $J$ Neurosurg 2009;110:446-456.

14. Biousse V, Bruce BB, Newman NJ. Update on the pathophysiology and management of idiopathic intracranial hypertension. J Neurol Neurosurg Psychiatry 2012;83(5):488494.

15. Corbett JJ, Mehta MP. Cerebrospinal fluid pressure in normal obese subjects and patients with pseudotumor cerebri. Neurol 1983;33:1386-1388.

16. Johnston I, Paterson A, Besser M. The treatment of benign intracranial hypertension: a review of 134 cases. Surg Neurol 1981;16:218-224.

17. Mollan SP, Markey KA, Benzimra JD, Jacks A, Matthews TD, Burdonet MA al. A practical approach to diagnosis, assessment and management of idiopathic intracranial hypertension. Pract Neurol 2014;14:380-390. 
18. Tibussek D, Distelmaier F, von Kries R, Mayatepek E. Pseudotumor Cerebri in Childhood and Adolescence -Results of a Germany-wide ESPED-survey. Klin Padiatr 2013;225:81-85.

19. Friedman DI, Liu GT, Digre KB. Revised diagnostic criteria for the pseudotumor cerebri syndrome in adults and children. Neurol 2013;81:1159-1165.

20. Distelmaier F, Mayatepek E, Tibussek D. Probable idiopathic intracranial hypertension in pre-pubertal children. Arch Dis Child 2008;93:356-357.

21. Vargas B. Normal pressure pseudotumour - is there evidence for a unique clinical entity? Neurol Reviews 2011;19(11):15

How to cite this article: Magesh $\mathrm{P}$, Kumar A. S, Prabhuraman K. Idiopathic intracranial hypertension or pseudotumour cerebri? The conundrum of nomenclature in the presence of normal cerebrospinal fluid pressures. Indian J Anat Surg Head Neck Brain 2018;4(4):106-110. 